\title{
Fractional Quantum Hall States and Jack Polynomials
}

\author{
B. Andrei Bernevigig ${ }^{1,2}$ and F.D.M. Haldane ${ }^{2}$ \\ ${ }^{1}$ Princeton Center for Theoretical Physics, Princeton, NJ 08544 and \\ 2 Department of Physics, Princeton University, Princeton, NJ 08544
}

(Dated: July 23, 2007)

\begin{abstract}
We describe an occupation-number-like picture of Fractional Quantum Hall (FQH) states in terms of polynomial wavefunctions characterized by a dominant occupation-number configuration. The bosonic variants of single-component abelian and non-abelian FQH states are modeled by Jacks (Jack symmetric polynomials), characterized by dominant occupation-number configurations satisfying a generalized Pauli principle. In a series of well-known Quantum Hall states, including the Laughlin, Read-Moore, and Read-Rezayi, the Jack polynomials naturally implement a "squeezing rule" that constrains allowed configurations to be restricted to those obtained by squeezing the dominant configuration. The Jacks describing uniform FQH states satisfy a highest-weight condition, and a clustering condition which can be generalized to describe quasiparticle states.
\end{abstract}

PACS numbers: 73.43.f, 11.25.Hf

The Laughlin wavefunction [1] has provided the key to understanding the physics of the fractional quantum Hall $(\mathrm{FQH})$ effect: it accurately models the simplest abelian FQH states and is the building block of model wavefunctions for more general states, both abelian, and (using cluster projections) non-abelian ones, such as the MooreRead 2] and Read-Rezayi [3] states. Apart from trivial (gaussian) factors which we will drop, such model wavefunctions are conformally-invariant multivariable polynomials $\psi\left(z_{1}, \ldots, z_{N}\right)$; despite their explicit availability, analytic calculations of correlation functions and other physical properties have not so far been possible because of the intractability of their expansions in the non-interacting basis of occupation number states (Slater determinants or monomials). As a result, quantitative study of these states has relied on exact diagonalization and other numerical methods [4].

The simplest physically-relevant model FQH states are antisymmetric polynomials, describing spin-polarized electrons in a partially-filled Landau level with no internal "pseudospin" degrees of freedom, but it is also useful to study symmetric (bosonic) FQH wavefunctions from which they are obtained by multiplication by odd powers of the Vandermonde determinant.

In this Letter, we describe a unified occupationbasis framework for the description of many model onecomponent FQH states in terms of the Jack symmetric polynomial(s) ("Jack(s)") [5]. The Jacks naturally implement a type of "generalized Pauli principle" on a generalization of Fock spaces for abelian and non-abelian fractional statistics [6]. We note that (bosonic) Laughlin, Moore-Read, and Read-Rezayi wavefunctions (as well as others, such as the state Simon et al. [7] have called the "Gaffnian") can be explicitly written as Jack symmetric polynomials, which have known (recursively defined) expansions in monomials (free boson occupation number states), and have rich algebraic properties. These uniform FQH condensate wavefunctions can be obtained by requiring that a Jack simultaneously obeys highestweight (HW, absence of quasiholes) and lowest-weight (LW, absence of quasiparticles) conditions.

Jacks $J_{\lambda}^{\alpha}(z)$ are symmetric polynomials in $z \equiv$ $\left\{z_{1}, z_{2}, \ldots, z_{N}\right\}$, labeled by a partition $\lambda$ with length $\ell_{\lambda} \leq N$, and a parameter $\alpha ; \lambda$ can be represented as a (bosonic) occupation-number configuration $n(\lambda)=$ $\left\{n_{m}(\lambda), m=0,1,2, \ldots\right\}$ of each of the lowest Landau level (LLL) orbitals with angular momentum $L_{z}=m \hbar$ (see Fig [1), where, for $m>0, n_{m}(\lambda)$ is the multiplicity of $m$ in $\lambda$. When $\alpha \rightarrow \infty, J_{\lambda}^{\alpha} \rightarrow m_{\lambda}$, which is the monomial wavefunction of the free boson state with occupationnumber configuration $n(\lambda)$; a key property of the Jack $J_{\lambda}^{\alpha}$ is that its expansion in terms of monomials only contains terms $m_{\mu}$ where $\mu \leq \lambda$, where $\mu<\lambda$ means the partition $\mu$ is dominated by $\lambda[5]$. Jacks are also eigenstates of a Laplace-Beltrami operator $\mathcal{H}_{\mathrm{LB}}(\alpha)$ given by

$$
\sum_{i}\left(z_{i} \frac{\partial}{\partial z_{i}}\right)^{2}+\frac{1}{\alpha} \sum_{i<j} \frac{z_{i}+z_{j}}{z_{i}-z_{j}}\left(z_{i} \frac{\partial}{\partial z_{i}}-z_{j} \frac{\partial}{\partial z_{j}}\right) .
$$

We note that the bosonic Laughlin state $\psi_{\mathrm{L}}^{(r)}$ at filling $\nu=1 / r, r$ even, is a Jack polynomial:

$$
\psi_{\mathrm{L}}^{(r)}=\prod_{i<j}^{N}\left(z_{i}-z_{j}\right)^{r}=J_{\lambda^{0}(1, r)}^{\alpha_{1, r}}(z), \quad \alpha_{k, r} \equiv-\frac{k+1}{r-1},
$$

which is the $k=1$ case of a Jack defined for any positive integer $k$ so that $N=k \bar{N}$, and $n_{m}\left(\lambda^{0}(k, r)\right)=k$ for $m=(j-1) r, j=1,2, \ldots, \bar{N}$, with $n_{m}=0$ otherwise. Note that $\lambda^{0}(k, r)$ is the " $(k, r, N)$-admissible" partition [8] that minimizes $|\lambda| \equiv M \equiv \sum_{m} m n_{m}$ at fixed $N(\lambda$ is "( $k, r, N)$-admissible" if $n(\lambda)$ obeys a "generalized Pauli principle" where, for all $m \geq 0, \sum_{j=1}^{r} n_{m+j-1} \leq k$, so $r$ consecutive "orbitals" contain no more than $k$ particles). Note that here the Jack parameter $\alpha_{k, r}$ is a negative rational; study of symmetric Jacks of this type was recently 
initiated in Ref. [8]. Earlier work generally assumes $\alpha$ is a positive real (Jacks with real $\alpha>0$ and unrestricted $\lambda$ occur in the solution of the integrable Calogero-Sutherland model[9]). Non-symmetric Jack-polynomials can also describe spin-FQHE wavefunctions such as Halperin and Haldane-Rezayi [10].

It is straightforward to see that $\psi_{\mathrm{L}}^{(r)}$ is a Jack: it has the obvious property that it is annihilated by operators

$$
D_{i}^{L, r}=\frac{\partial}{\partial z_{i}}-r \sum_{j(\neq i)}^{\prime} \frac{1}{z_{i}-z_{j}} ; \quad D_{i}^{L, r} \psi_{\mathrm{L}}^{(r)}=0 .
$$

It is then also annihilated by the combination $\sum_{i} z_{i} D_{i}^{L, 1} z_{i} D_{i}^{L, r}$, which is equal to $\mathcal{H}_{\mathrm{LB}}\left(\alpha_{1, r}\right)$ minus a constant (found by direct calculation to be $\frac{1}{12} r N(N-$ 1) $(N+1+3 r(N-1))$, so $\psi^{(r)}$ is an eigenstate of $\mathcal{H}_{\mathrm{LB}}\left(\alpha_{1, r}\right)$. It is now easy to identify the dominant configuration $n\left(\lambda_{1, r}^{0}\right)$, and verify that the eigenstate is nondegenerate, confirming Eq.(2). For $r=2$, this also follows implicitly from Ref. [8] where it was shown that the set of Jacks with parameter $\alpha_{k, 2}$ and $(k, 2, N)$-admissible $\lambda$ are a basis for the space of symmetric polynomials that vanish when $k+1$ variables $z_{i}$ coincide. The space of symmetric polynomials space can be divided into subspaces of fixed $M=|\lambda|$, and for $|\lambda|=\lambda_{k, r}^{0}$, there is a single $(k, r, N)$-admissible partition, so a polynomial with the appropriate properties is unique, and must be a Jack.

It is useful to identify the "dominance rule" (a partial ordering of partitions $\lambda>\mu$ ) with the "squeezing rule" 9$]$ that connects configurations $n(\lambda) \rightarrow n(\mu)$ : "squeezing" is a two-particle operation that moves a particle from orbital $m_{1}$ to $m_{1}^{\prime}$ and another from $m_{2}$ to $m_{2}^{\prime}$, where $m_{1}<m_{1}^{\prime} \leq m_{2}^{\prime}<m_{2}$, and $m_{1}+m_{2}=m_{1}^{\prime}+m_{2}^{\prime}$; $\lambda>\mu$ if $n(\mu)$ can be derived from $n(\lambda)$ by a sequence of "squeezings" (see Fig 1). This means that when model FQH wavefunctions equivalent to Jacks are expanded in basis of occupation number states, only configurations obtained by "squeezing"' from a dominant configuration will be present (this crucial property persists in fermionic model FQH wavefunctions given by the product of a Jack with a power of the Vandermonde determinant).

Jacks can be normalized so that

$$
J_{\lambda}^{\alpha}=m_{\lambda}+\sum_{\mu<\lambda} v_{\lambda \mu}(\alpha) m_{\mu} .
$$

The coefficients $v_{\lambda \mu}(\alpha)$ are (recursively) known [11]; they are finite and real positive for real $\alpha>0$, and are holomorphic functions of $\alpha$ except for poles at a $(\lambda, \mu)$ dependent set of negative rational values [8]. Feigin et.al. [8] proved that for the $(k, r, N)$-admissible partitions, $v_{\lambda \mu}(\alpha)$ is analytic at $\alpha_{k, r}$, and the set of admissible Jacks with this parameter form a basis of a differential ideal $I_{N}^{k, r}$ in the space of symmetric polynomials. This requires that $(k+1)$ and $(r-1)$ (but not necessarily $k$ and $r$ ) be coprime. For the case $r=2$, and $k$ integer, these polynomials are a basis for the $\nu=k / r=k / 2$ bosonic non-abelian

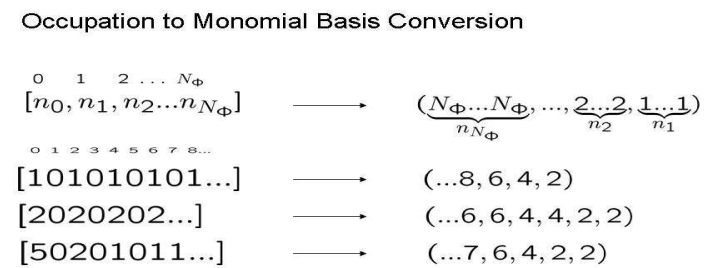

$\begin{array}{ll}\text { Squeezing Rule } & \\ {[100010001 \ldots] \longrightarrow} & {[\overrightarrow{010100001 \ldots]}} \\ & {[\overrightarrow{010010010 . . .]}} \\ & {[1000010 \hat{10 . . .]}}\end{array}$

FIG. 1: Occupation to monomial basis conversion and squeezing rule example

Read-Rezayi FQH states with quasiholes, with special cases $k=1$ (Laughlin state) and $k=2$ (Moore-Read state). By multiplying these wavefunctions by $\psi_{\mathrm{L}}^{(m)}$, this generalizes to the $\nu=k /(k m+2)$ Read-Rezayi states, and reproduces the "generalized Pauli principle" exclusion statistics structure found empirically in numerical studies by one of us [6].

The $\nu=1 / r$ Laughlin state is a Jack-polynomial with parameter $\alpha_{1, r}$ and $n(\lambda)=\left[10^{r-1} 10^{r-1} \ldots\right]$, where " $0^{r-1}$ " means a sequence of $r-1$ "empty orbitals". A basis of one-quasihole states can similarly be shown to be given by the Jack with $n(\lambda)=$ $\left[10^{r-1} 1 \ldots 0^{r-1} 10^{r} 10^{r-1} \ldots\right]$, where there is a single extra "empty orbital". These states all have different $M$, and hence are orthogonal, and form a multiplet. A linearlyindependent basis of two-quasihole state is given by Jacks with the same $\alpha$ and two extra "empty orbitals", in $n(\lambda)$. For example, at $r=2$, two such configurations $n(\lambda)$ and $n\left(\lambda^{\prime}\right)$ (with the same $M$ ) are $[10100010101 \ldots$. and $\left[10010100101 \ldots\right.$. . While $m_{\lambda}$ and $m_{\lambda^{\prime}}$ are orthogonal free-boson wavefunctions, the Jack FQH wavefunctions $J_{\lambda}^{\alpha_{1,2}}$ and $J_{\lambda^{\prime}}^{\alpha_{1,2}}$ are not orthogonal with respect to the usual quantum-mechanical scalar product. This highlights an important difference between the basis of "admissible Jacks" (with a "generalized Pauli principle") and the ordinary free-particle basis: the pure Jack wavefunctions are not eigenstates of a Hermitian Hamiltonian $\left(\mathcal{H}_{\mathrm{LB}}(\alpha)\right.$ is not Hermitian for finite $\left.\alpha\right)$, and are linearlyindependent but not orthogonal. (In contrast, Jacks with $\alpha$ real positive (and unrestricted $\lambda$ ) are orthogonal with respect to a combinatorically-motivated scalar product [5] and also as Calogero-Sutherland model wavefunctions.)

Partitions $\lambda$ can be classified by $\lambda_{1}$, their largest part. When $J_{\lambda}^{\alpha}$ is expanded in occupation-number states (monomials), no orbital with $m>\lambda_{1}$ is occupied, and Jacks with $\lambda_{1} \leq N_{\Phi}$ form a basis of FQH states on a sphere surrounding a monopole with charge $N_{\Phi}[4]$. Uniform states on the sphere satisfy conditions $L^{+} \psi=0$ (highest weight, HW) and $L^{-} \psi=0$ (lowest weight, LW) 
where $L^{+}=E_{0}$, and $L^{-}=N_{\Phi} Z-E_{2}$, where $Z \equiv \sum_{i} z_{i}$, and $E_{n}=\sum_{i} z_{i}^{n} \partial / \partial z_{i}$. When both conditions are satisfied, $E_{1} \psi \equiv M \psi=\frac{1}{2} N N_{\Phi} \psi$. It is very instructive to find the conditions for a Jack to satisfy the HW condition, $E_{0} J_{\lambda}^{\alpha}=0$. The action of $E_{0}$ on a Jack can be obtained from a formula due to Lassalle [12]: using the property that for real $\alpha>0$, all the $v_{\lambda}(\alpha)$ are real positive [5], the HW condition can only be satisfied for real $\alpha<0$. Another condition we find is that $n_{0} \equiv N-\ell_{\lambda}>0$ (nonzero occupancy of the $m=0$ "orbital"). We then find a necessary (but not sufficient) condition is

$$
N-\ell_{\lambda}+1+\alpha\left(\lambda_{\ell}-1\right)=0,
$$

where $\lambda_{\ell}$ is the smallest (non-zero) part in $\lambda$. This imposes the following two conditions: $(i) \alpha$ is a negative rational, which we can choose to write as $-(k+1) /(r-1)$, with $(k+1)$ and $(r-1)$ both positive, and relatively prime; $(i i) \lambda_{\ell}=(r-1) s+1$, and $n_{0}=(k+1) s-1$, where $s>0$ is a positive integer. The remaining HW conditions require that all parts in $\lambda$ have multiplicity $k$, so that $n(\lambda)=\left[n_{0} 0^{s(r-1)} k 0^{r-1} k 0^{r-1} k \ldots ..\right]$, (i.e, the $(k, r, N)$-admissibility condition is satisfied as an equality for orbitals $m \geq \lambda_{\ell}$ ). The case $s=1$ gives the FQH ground states which also obey the LW condition, with filling factor $\nu=k / r$, while the cases $s>1$ are intimately related to what we interpret as the quasiparticle (not quasihole) excitations of these $\nu=k / r$ FQH states, where $r=2$ corresponds to the bosonic Laughlin/MooreRead/Read-Rezayi sequence (see Fig_2).

We will describe the quasiparticle construction elsewhere [13], but note that when it is applied to the case $k=1, r=2(\nu=1 / 2$ bosonic Laughlin state $)$, it reproduces the model quasiparticle state given by Jain's projective construction [14]. The above derivation very simply reproduces the admissibility conditions found in Ref. [8], and shows that the $(k, r, N)$-admissible Jacks that minimize $M$ at fixed $N=k \bar{N}$ are the only pure Jacks that are acceptable FQH wavefunctions. We also remark that the construction of the $s>1$ sequence may prove useful [13] in connection with an open mathematical problem (the Cayley-Sylvester problem of classifying polynomials with coincident roots).

We now turn our attention to the Moore-Read state 2]. It was introduced as a model for the observed $\nu=5 / 2$ spin-polarized FQH $(\nu=1 / 2$ in the second LL) and is the $m=1$ case of the $\nu=2 /(2 m+2))$ state

$$
\Psi_{\mathrm{MR}}^{m}=\prod_{i<j}\left(z_{i}-z_{j}\right)^{m+1} \operatorname{Pf}\left(\frac{1}{z_{i}-z_{j}}\right),
$$

where $\Psi_{\mathrm{MR}}^{0}$ is a $\nu=1 \mathrm{FQH}$ state of bosons at $\nu=k / r$ where $k=r=2$ : The exclusion statistics picture of this bosonic state is $n\left(\lambda^{0}(2,2)\right)=[20202 \ldots]$ [6], or (the highest density state with) not more than 2 particles in 2 consecutive orbitals. It was initially defined as the correlation function of an Ising Majorana field $\psi_{(2,1)}=\psi(z)$

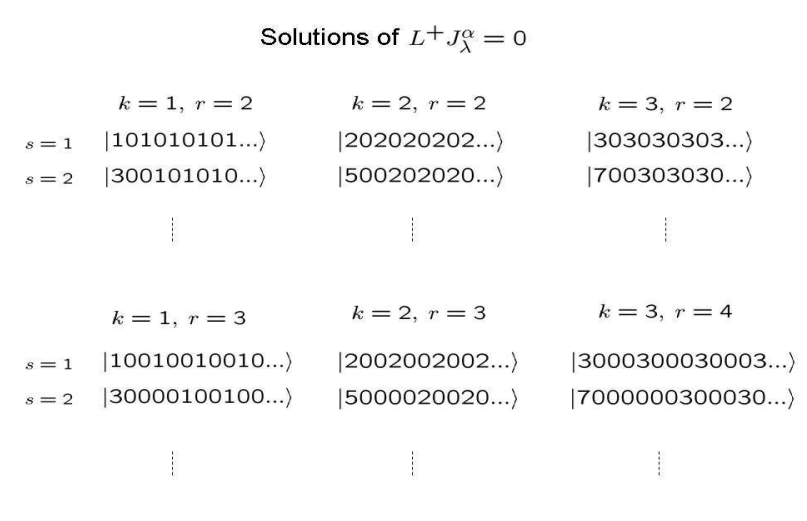

FIG. 2: Solutions to $L^{+} J_{\lambda}^{\alpha}=$ are parametrized by one integer, $s>0$. Only $s=1$ states are highest weight states on the sphere, and satisfy the clustering property that they vanish as the $r$ 'th power of the distance between $k+1$-particles. Other states, related to quasiparticles, satisfy generalized clustering conditions to be described in a future paper [13]

with scaling dimension $h_{2,1}=\frac{1}{2}$ in the minimal model $M(4,3)$ with $c=\frac{1}{2}$ (small indices label degenerate fields in CFT). The correlation functions of a field $\psi_{(m, n)}$ satisfy an $n m$ 'th-order differential equation. This allows us to define a set of $N$ annihilation operators. For the Pfaffian $\left\langle\psi\left(z_{1}\right) \psi\left(z_{2}\right) \ldots \psi\left(z_{N}\right)\right\rangle=\operatorname{Pf}\left(\frac{1}{z_{i}-z_{j}}\right)$ the annihilation operators are [15]:

$$
D_{i}^{\mathrm{Pf}}=\frac{\partial^{2}}{\partial z_{i}^{2}}-\sum_{j \neq i} \frac{A_{2,1}}{z_{i}-z_{j}} \frac{\partial}{\partial z_{j}}-\sum_{j \neq i} \frac{B_{2,1}}{\left(z_{i}-z_{j}\right)^{2}} ;
$$

where $A_{2,1}=2\left(2 h_{2,1}+1\right) / 3$ and $B_{2,1}=h_{2,1} A_{2,1}$. The Pfaffian satisfies $D_{i}^{\mathrm{Pf}} \operatorname{Pf}\left(\frac{1}{z_{i}-z_{j}}\right)=0$. According to the general prescription for obtaining Quantum Hall wavefunctions out of CFT correlators, the first bosonic MooreRead state $\psi_{\mathrm{MR}}^{0}$ is obtained by multiplying the Pfaffian by a Vandermonde factor: $\psi_{\mathrm{L}}^{(1)}$. It is straightforward to transform $D_{i}^{\mathrm{Pf}}$ to obtain related operators $D_{i}^{\mathrm{MR}}$ that annihilate $\psi_{\mathrm{MR}}$, and show that $\sum_{i} D_{i}^{\mathrm{MR}}$ is $\mathcal{H}_{\mathrm{LB}}(-3)$ plus a constant (found by direct computation to be $\left.-N\left(16-18 N+5 N^{2}\right) / 18\right)$, which confirms that $\psi_{\mathrm{MR}}^{0}$ $=J_{\lambda^{0}(2,2)}^{-3}$.

The $\nu=k / 2$ bosonic Read-Rezayi (RR) states [3] are " $Z_{k}$ parafermion states". The first RR state is related to the $Z_{3}$ Potts model [16] and is annihilated by a third order differential operator. The dominant configuration this state is $n\left(\lambda^{0}(3,2)\right)=[3030303 \ldots]$, or (the highest density state with) not more than 3 particles in 2 consecutive orbitals. The $Z_{3}$ parafermion quantum Hall state is a single Jack and diagonalizes the second-order LaplaceBeltrami operator. The $\operatorname{RR~} Z_{k}$ sequence is $\psi_{R R}^{0}(z)=$ $J_{\lambda^{0}(k, 2)}^{\alpha(k, 2)}(z)$.

A bosonic state at $\nu=2 / 3$ (or a fermionic one with $\nu=2 / 5$ ) has been referred to as a "Gaffnian" 7]. 
The dominant configuration of the bosonic state $\psi_{\mathrm{G}}^{0}$ is $n\left(\lambda^{0}(2,3)\right)=[2002002002 \ldots]$, or the highest density $(2,3)$ state. We find this state is annihilated by (7) with $h_{2,1}=3 / 4$, as expected, as the wavefunction of this state is also the correlation function of a minimal CFT $M(5,3)$ field $\psi_{(2,1)}$ with this scaling dimension [7], and we identify $\psi_{\mathrm{G}}^{0}(z)$ as the $(2,3)$ vacuum Jack $J_{\lambda^{0}(2,3)}^{\alpha_{2,3}}(z)$.

Instead of using the differential equations that they satisfy, it is easier to identify the FQH states with Jacks from their clustering properties. Information on how the Jacks vanish as $k+1$ coordinates coincide is needed: we verified that, for any $(k, r, N)$-admissible $\lambda$, if $z_{1}=z_{2}=$ $\ldots=z_{k}=Z$, that $J_{\lambda}^{\alpha_{k, r}}(z)$ has a factor $\prod_{i=k+1}^{N}\left(Z-z_{i}\right)^{r}$, showing how it vanishes as a cluster of $k+1$ coincident coordinates is formed. This agrees with the known properties of the bosonic Laughlin/Moore-Read/Read-Rezayi states $(k \geq 1, r=2)$, as well as the "Gaffnian" $(k=2$, $r=3)$. For the case of the FQH ground states, where $\lambda$ $=\lambda_{k, r}^{0}$, the Jacks satisfy a stronger clustering property that relates $N$ - and $(N+k)$-particle states: for $\{z\} \equiv$ $\left\{z_{1}, \ldots, z_{N}\right\}$

$$
\prod_{i=1}^{N}\left(Z-z_{i}\right)^{r} J_{\lambda^{0}(k, r)}^{\alpha_{k, r}}(\{z\})=J_{\lambda^{0}(k, r)}^{\alpha_{k, r}}(\{z\}, Z, \ldots, Z),
$$

where on the RHS, $z_{i}=Z$ for $i=N+1, \ldots, N+k$. As a corollary, when a $(k, r)$ Jack FQH ground state is fully $k$-clustered, i.e., $z_{k i-j} \rightarrow Z_{i}$ for $i=1, \ldots, \bar{N}$ and $j=1, \ldots, k$, it becomes a Laughlin state in the cluster coordinates:

$$
J_{\lambda^{0}(k, r)}^{\alpha_{k, r}}(z) \rightarrow \psi_{L}^{(k r)}\left(Z_{i}\right) .
$$

These properties show that, for these model FQH states, removing a cluster of $k$ particles at a point $Z$ is exactly equivalent to inserting $r$ flux quanta (or vortices) at that point (as is well-known in the $k=1$ Laughlin case, and implicit in the Read-Rezayi construction of the parafermion states as symmetrizations over distinct pairs of Laughlin-like clusters of particles [3] ).

As is obvious from their clustering property, the $(k, r, N)$-admissible Jacks also have the property that a $(k+1)$-cluster of particles cannot have relative angular momentum less than $r$, and hence are simultaneous null states of Hermitian operators $\hat{H}_{r-1}^{(k+1)}$, which are the $(k+1)$-body generalizations [7] of two-body Hamiltonians $\hat{H}_{r=1}^{(2)}$ where the only non-zero two-body pseudopotentials [4] are $V_{m}>0$ for $m \leq r-1$ However, for $k>1, r>3$ (and $(k+1)$ and $(r-1)$ relatively prime), the number of linearly-independent null states of $\hat{H}_{r-1}^{(k+1)}$ is larger than the set of $(k, r, N)$-admissible Jacks, and in particular, the homogeneous $\nu=k / r \mathrm{FQH}$ state with $\lambda$ $=\lambda^{0}(k, r)$ is not in general unique, as seen in Table I of 17]. For example, for $(k, r)=(3,4)$, (spinless boson states with $\left.N=3 \bar{N}, N_{\Phi}=4(\bar{N}-1)\right)$, the $(3,4, N)$-admissible Jack with $\lambda=\lambda^{0}(3,4)$ is a zero-mode eigenstate of $\hat{H}_{3}^{(4)}$ but is not unique. We did not find any other local Hermitian $n$-body pseudopotential operators that could be added to $\hat{H}_{3}^{(4)}$ to make this Jack a unique null state, so it remains unclear how to define the $k>1, r>3$ Jack FQH states as unique null states of a model Hamiltonian (although requiring them to also be eigenstates of the non-Hermitian operator $\mathcal{H}_{\mathrm{LB}}$ does make them unique). Mathematically, they are related to correlation functions of primary fields of non-unitary minimal-sequence conformal field theories [7, 8].

In conclusion, we have identified a number of model bosonic $\mathrm{FQH}$ ground states at $\nu=k / r$ (with $k+1$ and $r-1$ relatively prime) with a set of special Jack symmetric polynomials, whose expansion in monomials (free-particle occupation-number states) is (recursively) known. The FQH states described here, being single Jacks, are eigenstates of a multiplet of $N$ mutually commuting, higher derivative many-body operators (Sekiguchi operators) [18, 19], of which the first is $E_{1}=$ $M$, the total momentum, and the second is $\mathcal{H}_{L B}(\alpha)$. We obtained the $(k, r, N)$-admissible partitions [8] as a special case of the highest weight conditions on the Jacks that generalizes to a $(k, r, s, N)$-admissibility to include quasiparticle states [13]. It may be hoped that the identification of such larger underlying algebraic properties of the conformally-invariant model FQH state will lead to the development of analytic (as opposed to numerical) techniques for calculation of their correlation functions an other properties.

This work was supported in part by the U.S. National Science Foundation (under MRSEC Grant No. DMR0213706 at the Princeton Center for Complex Materials).

[1] R.B. Laughlin, Phys. Rev. Lett. 50, 1395 (1983).

[2] G. Moore and N. Read, Nucl. Phys. B 360, 362 (1991).

[3] N. Read and E. Rezayi, Phys. Rev. B 59, 8084 (1999).

[4] F.D.M. Haldane, Phys. Rev. Lett. 51, 605 (1983).

[5] R.P. Stanley, Adv.Math. 77, 76 (1989).

[6] F.D.M. Haldane, Bull. Am. Phys. Soc. 51, 633 (2006).

[7] S.H. Simon, E.H. Rezayi, N.R. Cooper, and I. Berdnikov, PRB 75, 075317 (2007).

[8] B. Feigin, M. Jimbo, T. Miwa, and E. Mukhin, Int. Math. Res. Not. 23, 1223 (2002).

[9] B. Sutherland, Phys. Rev. A 4, 2019 (1971).

[10] M. Kasatani and V. Pasquier, cond-mat/0608160

[11] L. Lapointe, A. Lascoux, and J. Morse, Elec. J. Comb. 7, 1 (2000).

[12] M. Lassalle, J. Func. Anal. 158, 289 (1998).

[13] B.A. Bernevig and F.D.M. Haldane, in preparation.

[14] J.K. Jain, Phys. Rev. Lett. 63, 199 (1989).

[15] A.A. Belavin, A.M. Polyakov, and A.B. Zamolodchikov, Nucl. Phys. B 241, 333 (1984).

[16] V.S. Dotsenko, Nucl. Phys. B 235, 54 (1984).

[17] S.H. Simon, E.H. Rezayi, and N.R. Cooper, PRB 75, 075318 (2007).

[18] T. Osima and H. Sekiguchi, J. Math. Sci. Univ. Tokyo 2, 
1 (1995)

[19] D. Bernard, M. Gaudin, F.D.M. Haldane, and

V. Pasquier, J. Phys. A: Math. Gen 26, 5219 (1993). 